ScIDice

\section{Influence of Clinical and Pathological Characteristics of OSCC on Disease Free and Overall Survival - A Single Institute Study}

\section{International Journal of Dentistry and Oral Science (IJDOS) \\ ISSN: $2377-8075$}

Research Article

Sunil Akula ${ }^{1}$, Mahathi Neralla ${ }^{2 *}$, Rinku George ${ }^{3}$

${ }^{1}$ Fellow, Saveetha Oral Cancer Institute, Department of Oral and Maxillofacial Surgery, Saveetha Dental College, 162, Poonamallee High Road, Velappanchavadi, Chennai, Tamil Nadu, India.

${ }^{2}$ Reader, Saveetha Oral Cancer Institute, Department of Oral and Maxillofacial Surgery, Saveetha Dental College, 162, Poonamallee High Road, Velappanchavadi, Chennai, Tamil Nadu, India.

${ }^{3}$ Professor, Saveetha Oral Cancer Institute, Department of Oral and Maxillofacial Surgery, Saveetha Dental College, 162, Poonamallee High Road, Velappanchavadi, Chennai, Tamil Nadu, India.

\title{
Abstract
}

Objective of our study: To find out the significance of clinical and pathological characteristics on the survival rate and recurrence in the patients treated at our hospital.

Materials and methods: Retrospective analysis of post-operative histopathology reports of patients operated in our college from 2015-2017 were analysed and all the data related to margins and other necessary clinical and pathological data were noted. We classified margins classified into clear $(\geq 5 \mathrm{~mm})$, mildly dysplastic, moderately dysplastic, severely dysplastic and inadequate margins/positive margins by the pathologist as per previous available literature. All patients were operated on with the primary objective of achieving a macroscopic clearance of $1 \mathrm{~cm}$. Postoperative radiotherapy was advised in indicated cases. Known clinical and pathological prognostic indicators, in addition to the condition of surgical margins, were analysed to find out if they were predictive of overall survival and disease-free survival using the chi- square test. The survival time was calculated using Kaplan-Meier survival curve. The log rank test was used to detect the differences for survival time. We compared this data with the previously available literature.

Results: Overall median survival was 34.96 months and median disease-free survival is 24.83 months. Histological grade and stage at diagnosis was identified as having a statistically significant effect on survival ( $\mathrm{p}$ value $=0.001$ and 0.033 ) respectively. All the other associations between age, gender, subsite, histological variant of disease, adjuvant therapy administration, status of surgical margins and disease free and overall survival was not found to be significant.

Outcome and Conclusion: Histological grade of the disease and stage at presentation seem to be important factors in determining disease free and overall survival stressing the importance of early detection and treatment of oral cancer to improve a patient's chance of success in terms of long term quality of life without recurrence.

Keywords: Overall Survival (OS); Disease Free Survival (DFS); Local Recurrence (LR); Surgical Margins.

\section{Introduction}

Oropharyngeal squamous cell carcinoma is the sixth most common malignancy in the world, but incidence and mortality rate have regional variations. These variations are observed due the variation in the prevalence of risk factor usage like tobacco [1].

Even with improvement in outcomes over the last decade oral squamous cell carcinoma (OSCC) still has a relatively unfavourable prognosis with 5-year overall and disease-free survival estimated to be $56 \%$ and $58 \%$, respectively [2].

Obtaining a clear surgical margin at the time of surgery is of utmost importance. This is proven to influence the postoperative course of the disease in patients operated for oral squamous cell carcinoma. There have been studies that advise on the importance

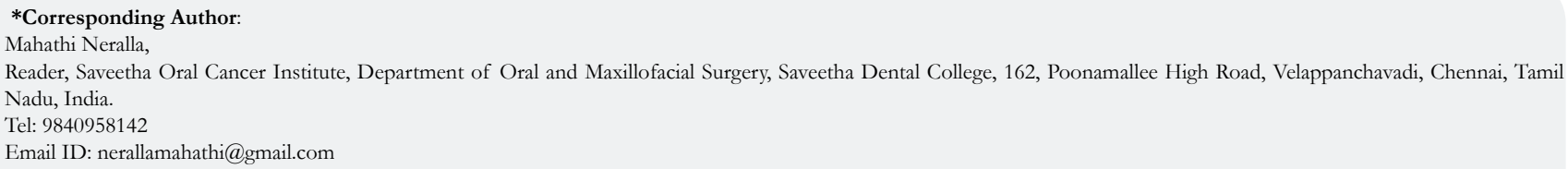

Received: March 15, 2021

Accepted: April 02, 2021

Published: April 06, 2021

Citation: Sunil Akula, Mahathi Neralla, Rinku George. Influence of Clinical and Pathological Characteristics of OSCC on Disease Free and Overall Survival - A Single Institute Study. Int J Dentistry Oral Sci. 2021;08(04):2205-2213. doi: http://dx.doi.org/10.19070/2377-8075-21000436

Copyright: Mahathi Neralla ${ }^{\circ} 2021$. This is an open-access article distributed under the terms of the Creative Commons Attribution License, which permits unrestricted use, distribution and reproduction in any medium, provided the original author and source are credited. 
of postoperative radiotherapy to negate the adverse impact of obtaining a positive or dysplastic margin but still the local recurrence rates and overall survival rate is decreased when compared with patients for whom clear margin was obtained [3].

The primary objective of cancer resection is to obtain clear margin, that is, to obtain $\mathrm{R} 0$ resection. There have been many studies that prove the adverse impact of a positive surgical margin. Involved surgical margins increased the risk of death at 5 years by $90 \%$ was concurred from the study by AbdulazizBinahmed et al [4]. The same author in his study article says that local recurrence also increases by a factor of two or more when the presence of microscopic tumor is deducted at or close to the resected margin [4]. Hence the surgeon's primary objective is to obtain clear margin at any cost during surgery. But achieving histologically adequate margins alone does not translate into increased overall survival especially disease free survival [5]. The other factors that influence survival along with margin status are T-stage of the tumor, $\mathrm{N}$-stage, histopathologicalfeatures including tumor thickness and the pattern of invasion [4]. For example, tumor thickness for T1 tongue squamous cell carcinoma (SCC) has been strongly associated with an increased propensity for lymph node metastasis but is not a predictor for LR [5]. Hence when conducting a study, we should be able to assess the independent effect of a variable on the outcome that is to be studied, or rather we should be able to formulate a study design that enables us to do so. Another histological characteristic that influences or predicts local recurrence (LR) and overall survival (OS) is the pattern of tumor invasion (POI). Pattern of tumor invasion (POI) is the way in which cancer infiltrates tissue at the tumor/host interface. Neoplasia that infiltrates in a widely dispersed manner is more aggressive than those growing in a bulky pushing fashion. POI alone, and as part of other scoring systems, helps to predict LR and decreased overall survival (OS) [5].

Our aim was to understand the extent of influence of clinical and pathological features on the overall and disease free survival rates. We looked into our database of cases to assess the influence of age, gender, subsite, histological differentiation of the tumor, $\mathrm{T}$ stage, $\mathrm{N}$ stage, overall stage, and the effect of adjuvant treatment on overall survival and disease free survival among the many factors that has been quoted in the literature to have an effect on overall survival (OS) and disease free survival (DFS) or local recurrence (LR).

\section{Materials and Methods}

Our methodology mimics few other studies that have been done in the past, with few variations according to the data we collected from the patients treated in our Institute. This Retrospective study included patients who were treated at the Department of Oral Oncology and Reconstructive Services of a Private Dental College, Chennai. Medical records of patients operated in our Institute from June 2015- April 2020 were reviewed. All the required data like demographic details, age, sex and type of surgery and other required data for the study being performed were noted.

All the patients who walk into our Institute are evaluated first and TNM staging done according to the AJCC 7th edition. Patients are worked up for surgery if their disease status allows the lesion to be operated. The primary aim of the surgery is to obtain
$1-1.5 \mathrm{~cm}$ macroscopic margin of clearance.

\section{Inclusion criteria}

1. Patients with histologically proven squamous cell carcinoma of the oral cavity.

2. Patients not previously treated elsewhere.

3. Patients for whom surgery was the first option and was done as their initial treatment.

\section{Exclusion criteria}

1. Patients not fit for surgery and presence of other contraindications for surgery (inoperable or unresectabletumors ).

2. Presence of distant metastasis or local recurrence at the time of admission

3. Presence of other simultaneous primary tumors or conditions that precludes surgery.

4. Patients managed with preoperative radiotherapy and neoadjuvant chemotherapy.

Surgery was the preferred treatment modality in all presenting with stage I and II. For patients presenting with stage III and IV the decision to do surgery first was decided after a tumor board meet. The decision to do or not to do neck dissection and the type of neck dissection, either selective neck dissection (SOHND; level I, II, III) or mRND (level I, II, III, IV, V) was performed, depending on neck involvement and tumor size, NCCN guidelines and institutional protocol. The aim of the primary tumor resection was to achieve three-dimensional macroscopic clearance. In-continuity primary resections were done based on operator preference with importance given mainly to three-dimensional clearance. As a routine, intraoperative frozen section is done and any close margin is revised further, in our Institute. Postoperatively, the resected specimen was sent for biopsy for detailed examination. The biopsy specimens were labelled with neck levels and sublevels of dissection and were immersed in $10 \%$ buffered formalin and sent for examination.

After the surgery, post-operative histopathology records are reviewed and the margins were classified into clear $(\geq 5 \mathrm{~mm})$, mildly dysplastic, moderately dysplastic, severely dysplastic and inadequate margins/positive margins by the pathologist.

Postoperative radiotherapy was advised according to Institutional protocol: when $\mathrm{T}$ stage is more, when lymph nodes are involved, any extracapsular spread, perineural invasion or positive margin, or if depth of invasion greater than $10 \mathrm{~mm}$. If a positive margin is obtained we first tried to revise it surgically if possible. An involved margin was defined as one where tumour was reported as present at the surgical resection margin, or within $1 \mathrm{~mm}$ of a resection margin, and therefore likely to be involved [3].

Radiotherapy was initiated within 4 to 6 weeks after surgery if necessary, according to the patient's condition. Follow up of the postoperatively was done once every 4 weeks for the first year and once every 2 months in the second year.

We retrospectively analysed all these data and collected them on an excel sheet, so statistical analysis could be done.

The Institutional Ethics Committee approved the study. The 
ethics committee number is SRB/SDC/FACULTY/20/OS$\mathrm{URG} / 01$

\section{Statistical methods}

Our measures of outcome were disease free survival and overall survival. All statistical analyses were performed with SPSS 20.0 version. The association of predictors with overall survival and disease free survival was done using chi- square test. The survival time was calculated using Kaplan-Meier survival curve. The log rank test was used to detect the differences for survival time. The $\mathrm{p}<0.05$ was considered significant.

\section{Results}

Of 95 patients, $71(74.73 \%)$ were men and $24(25.26 \%)$ were women; the number of patients with $<50$ age at diagnosis was $42(44.21 \%)$ and $>50$ was $53(55.78 \%)$. There were $22(23.15 \%)$ patients with clear margin, $12(12.63 \%)$ with positive margin, 34 (35.78\%) with mild dysplastic margin, 23 (24.21\%) with moderate dysplastic margin and $4(4.21 \%)$ with severe dysplastic margin. The basic characteristics of the patients are shown in Table 1. There were no significant differences among the patients with clear, positive or dysplastic margins in terms of age, sex, primary tumor location with regards to disease free and overall survival, but there was a significant difference when the stage of the disease was considered in terms of disease free and overall survival ( $p<0.05)$. Overall median survival was 34.96 months and median disease-free survival is 24.83 months.

\section{Subsites distribution}

According to the subsite, buccal mucosa and gingivobuccal sulcus lesion contributed to the majority of cases [36 (37.89\%)] followed by oral tongue and floor of the mouth lesions which accounted for $27(28.42 \%)$ and retromolartrigone (RMT) 17 (17.89\%) cases. The number of pure alveolus cases were $8(8.4 \%)$, palate and maxilla cases were $7(7.36 \%)$ respectively.

\section{Type of neck dissection}

Out of the 95 cases MRND was performed in 56 (58.94\%) of patients and SND was performed in $35(36.84 \%)$ of patients. In 4 cases $(4.21 \%)$ neck dissection was not performed.

\section{Neck stages distribution}

On clinical examination, $49(51.57 \%)$ had been staged to have nodal disease.

\section{Adjuvant therapy distribution}

$43(45.26 \%)$ patients received adjuvant therapy in the form of radiotherapy and chemotherapy and $52(54.73 \%)$ of them had not received either radiotherapy or chemotherapy.

\section{Influence of clinical and pathological characteristics on dis- ease free and overall survival}

\section{Age and Gender}

Overall survival: Out of 42 patients who are less than or equal to 50 years of age, $28(66.7 \%)$ of them are still alive and $14(33.3 \%)$ are dead with a median survival of 24.83 months. And out of 53 patients whose age is above 50 years, 38 are still alive and 15 (28.3 $\%$ ) are dead with a median survival of 34.96 months and with a similar overall survival of 34.96 months.

Out of 71 male patients 49 (69\%) are alive and $22(31.0 \%)$ have died with a median survival of 27.40 months and among $24 \mathrm{fe}-$ males, $17(70.8 \%)$ have died while $7(29.7 \%)$ are still alive with a median survival of 34.96 months. The median age for overall survival is 34.96 months.

The $\mathrm{p}$ values for age and gender in Kaplan-Meier analysis for overall survival was 0.549 and 0.630 respectively which are not significant (Fig 1 and 2).

\section{Disease free survival}

Out of 42 patients below or equal to 50 years of age, 19 (45. 2 $\%$ ) of them had disease free survival and 23 (54.8\%) of them had recurrence of disease with a median disease free survival of 22.26 months. Out of 53 patients above the age of 50 years, 34 (64.2\%) of them had disease free survival, while $19(35.8 \%)$ of them had recurrence with a median disease free survival of 33.83 months.

Out of 71 males, $41(57.7 \%)$ had disease free survival while 30 $(42.3 \%)$ had recurrence with a median disease free survival of 23.5 months and out of 24 females, $12(50 \%)$ had disease free survival and $12(50 \%)$ had recurrence of disease with a median disease free survival of 25.43 years. The overall median for disease free survival is 24.83 months.

The $\mathrm{p}$ values for age and gender in Kaplan-Meier analysis for disease free survival for age and gender were 0.112 and 0.974 respec-

Figure 1. Kaplan-Meier overall survival curves for the two age groups. There is a no significant difference between both age groups $(\mathrm{p}=\mathbf{0 . 5 4 9})$.

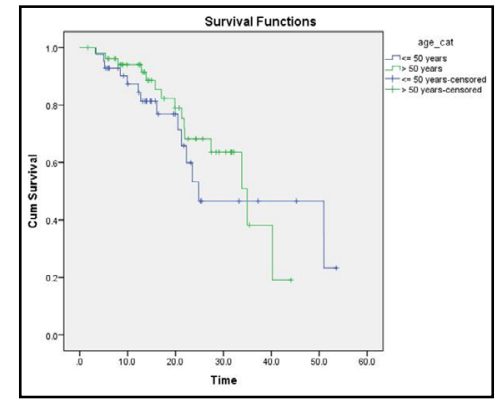


tively which are not significant (Fig 3 and 4).

\section{Surgical margin}

Overall survival: Out of the 22 patients with clear surgical margins, $17(77.3 \%)$ survived and $5(22.7 \%)$ died with a median survival of 34.96 months and out of 34 patients with mild dysplastic margins $24(70.6 \%)$ survived and $10(29.4 \%)$ died. Out of 23 patients with moderately dysplastic margins, 14 (60.9\%) survived and $9(39.1 \%)$ died with a median survival of 24.83 months and out of 4 patients with severely dysplastic margins, $3(75 \%)$ survived and 1 (25\%) died. When considering 12 cases with intraoperative inadequate margins or those margins which showed the presence of tumour cells, $8(66.7 \%)$ of them survived while $4(33.3 \%)$ died with a median survival of 50.96 months. The median overall survival is 34.967 months. The $\mathrm{p}$ value for surgical margins in Kaplan-Meier analysis for overall survival was 0.485 which is not significant (Fig 5).

Disease free survival: When the influence of surgical margins on disease free survival were considered, 22 patients had adequately clear margins out of which $15(68.2 \%)$ were disease free and $7(31.8 \%)$ had recurrence with a median disease free time of 34.96 months. Out of 34 patients with mildly dysplastic margins

Figure 2. Kaplan-Meier overall survival curves for gender (0 - Male; 1 - Female). There is a no significant difference between male and female $(\mathrm{p}=\mathbf{0 . 6 3 0})$.

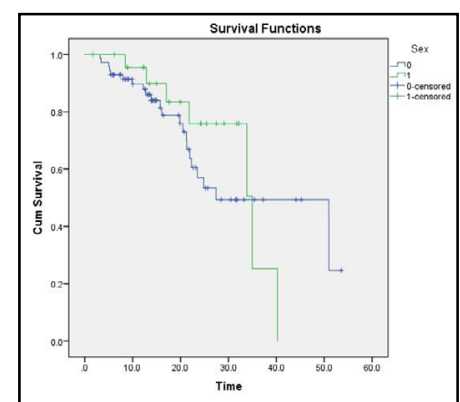

Figure 3. Kaplan-Meier disease free survival curves for the two age groups. There is a no significant difference between both age groups $(\mathrm{p}=\mathbf{0 . 9 7 4})$.

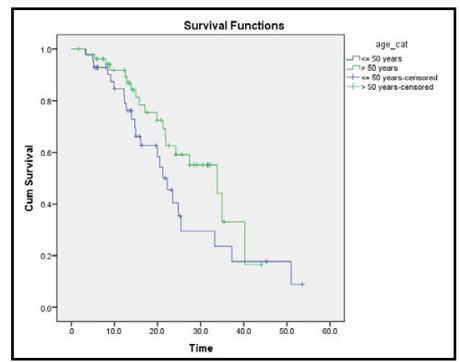

Figure 4. Kaplan-Meier disease free survival curves for gender (0- Male; 1- Female). There is a no significant difference between male and female $(\mathrm{p}=\mathbf{0 . 9 7 3})$.

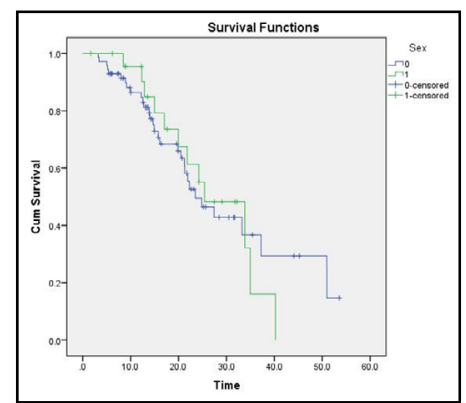

Figure 5. Kaplan-Meier overall survival curves for surgical margins. There is a no significant difference between the different margin status $(\mathrm{p}=0.485)$.

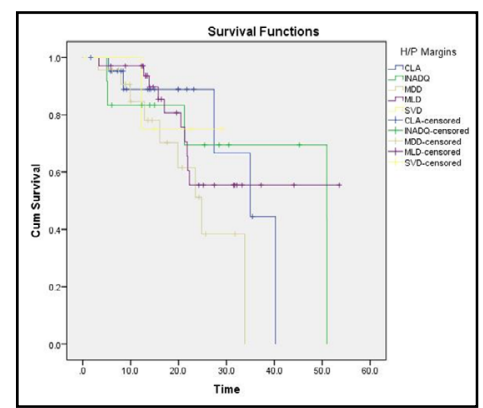


$19(55.9 \%)$ were free of the disease and $15(44.1 \%)$ had recurrence with a median disease free period of 24.23 months. In the same manner, out of 23 patients with moderately dysplastic margins and 4 patients with severely dysplastic margins, $13(56.5 \%)$ and $2(50 \%)$ had disease free survival respectively and $10(43.5$ $\%)$ and $2(50 \%)$ had recurrence of the disease respectively with a median disease free period of 23.50 months for moderately dysplastic margins and 14.93 for severely dysplastic surgical margins. Out of 12 patients with inadequate margins 4 (33.3\%) had disease free survival while $8(66.7 \%)$ of them had recurrence of the disease with a median disease free survival of 21.23 months. The $\mathrm{p}$ values for surgical margins in Kaplan-Meier analysis for disease free survival was 0.832 which is not significant (Fig 6).

\section{Subsite}

Overall survival: When subsite and overall survival were evaluated, out of 8 cases of alveolus $5(62.5 \%)$ are alive and $3(37.5 \%)$ are dead with a median survival of 34.96 months, out of 36 cases of buccal mucosa and gingivo-buccal sulcus, 22 (66.1\%) are alive while $14(8.9 \%)$ are dead with a median survival of 27.40 and out of 27 cases of oral tongue and floor of the mouth cases, 22 (81.5 $\%$ ) are alive while $5(18.5 \%)$ are dead. There were 7 cases of palate and maxilla out of which $5(71.4 \%)$ are alive while $1(28.6 \%)$ are dead and out of 17 cases of RMT, $12(70.6 \%)$ are alive and $5(29.4 \%)$ are dead with a median survival of 33.83 months. The overall median survival is 34.96 months. The $\mathrm{p}$ value for subsite in Kaplan-Meier analysis for disease free survival is 0.843 which is not significant (Fig 7).

Disease free survival: When subsite and disease free survival were assessed, out of 8 cases of alveolus $4(50 \%)$ are disease free and $4(50 \%)$ have a recurrence of disease with a median disease free survival of 34.96 years, out of 36 cases of buccal mucosa and gingivo-buccal sulcus, $17(47.2 \%)$ are disease free while 19 $(52.8 \%)$ have a recurrence of disease with a median disease free survival of 22.26 months and out of 27 cases of oral tongue and floor of the mouth, $18(66.7 \%)$ are disease free while $9(33.3 \%)$ have a recurrence of disease with a median disease free survival of 24.83 months. There are a total of 7 cases of palate and maxilla out of which $2(28.6 \%)$ are disease free while $5(71.4 \%)$ have a recurrence of disease with a median disease free survival of 19.96 months and out of 17 cases of RMT, 12 (70.6\%) are disease free and $5(29.4 \%)$ have a recurrence of disease with a median disease free survival of 33.83 years. The overall median for disease free survival is 24.83 months.

The $\mathrm{p}$ value for subsite in Kaplan-Meier analysis for disease free survival was 0.245 which is not significant (Fig 8).

\section{T stage, $\mathbf{N}$ stage and overall stage}

Overall survival: While considering T stage, out of 16 patients with T1 lesion, $15(93.98 \%)$ are alive while 1 (6.2\%) died, out of

Figure 6. Kaplan-Meier disease free survival curves for surgical margins. There is a no significant difference between the different margin status $(\mathrm{p}=0.832)$.

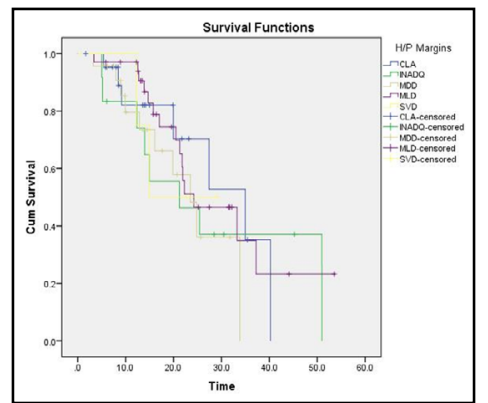

Figure 7. Kaplan-Meier overall survival curves for subsite. There is a no significant survival difference between the subsites( $\mathrm{p}=\mathbf{0 . 8 4 3 )}$.

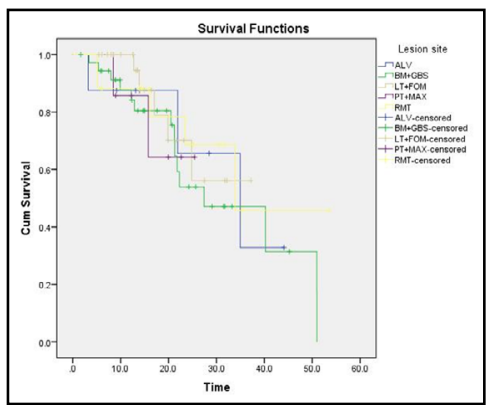

Figure 8. Kaplan-Meier disease free survival curves for subsite. There is a no significant disease free survival difference between the subsites $(\mathrm{p}=0.245)$.

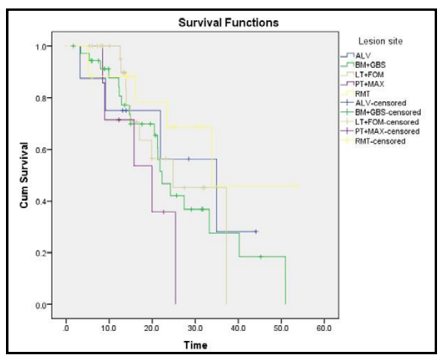


35 patients with T2 lesion, 25 (71.4\%) are alive while 10 (28.6\%) are dead with a median disease free survival of 33.83 months, out of 16 patients with T3 lesion, $9(56.2 \%)$ are alive while $7(4.8 \%)$ are dead with a median disease free survival of 21.23 months and out of 28 patients with T4 lesion, 17 (60.7\%) are alive while $11(39 . \%)$ are dead with a median disease free survival of 34.96 months $(\mathrm{P}$ value $=0.056)$.

While considering $\mathrm{N}$ stage, out of 46 cases with $\mathrm{N} 0$ status, 4 $(73.9 \%)$ are alive while $12(26.1 \%)$ are dead with a median survival of 50.96 months, out of 28 patients with N1 status, $21(75 \%)$ are alive while $7(25 \%)$ are dead with a median survival of 33.83 months and 21 patients with N2 status, 11 (52.4\%) are alive while $10(47.6 \%)$ are dead with a median survival of 27.40 months $(\mathrm{P}$ value $=0.596)$.

When overall stage was considered, out 11 patients with stage 1 disease, all $11(100 \%)$ are alive, out of 22 patients with stage 2 disease, $17(77 \%)$ are alive while $5(22.7 \%)$ are dead and out of 26 patients with stage 3 disease, $18(69.2 \%)$ are alive while $8(30.8 \%)$ are dead and out of 36 patients with stage 4 disease, $20(55.6 \%)$ are alive and $16(44.4 \%)$ are dead.

The $\mathrm{p}$ value for overall stage in Kaplan-Meier analysis for overall survival was 0.224 which is not significant.

The $\mathrm{p}$ value for overall survival when chi square test was done for the overall stage of disease was $0.033(\mathrm{p}<0.05)$ and is statistically significant (Fig 9-11).

Disease free survival: While considering $\mathrm{T}$ stage, out of $16 \mathrm{pa}-$ tients with T1 lesion, $13(81.2 \%)$ are disease free while $3(18.8 \%)$ had recurrence of disease with a median disease free survival of 37.23 months, out of 35 patients with T2 lesion, 19 (54.3\%) are disease free while $16(45.7 \%)$ had recurrence of disease with a median disease free survival of 22.26 months, out of 16 patients with T3 lesion, $9(56.2 \%)$ are disease free while $7(43.8 \%)$ had recurrence of disease with a median disease free survival of 21.23 months and out of 28 patients with T4 lesion, 12 (42.9\%) are alive while $16(57.1 \%)$ had recurrence of disease with a median disease free survival of 24.23 months. The overall median disease free survival is 24.83 months ( $\mathrm{p}$ value $=0.259)$.

While considering $\mathrm{N}$ stage, out of 46 cases with $\mathrm{N} 0$ status, 28 $(60.9 \%)$ are disease free while $18(39.1 \%)$ had recurrence of disease with a median disease free survival of 21.93 months, out of 28 patients with N1 status, $18(64.3 \%)$ are disease free while $10(35.7 \%)$ had recurrence of disease with a median disease free

Figure 9. Kaplan-Meier overall survival curves for the $T$ stage. There is a no significant difference between different $T$ stages $(\mathrm{p}=0.056)$.

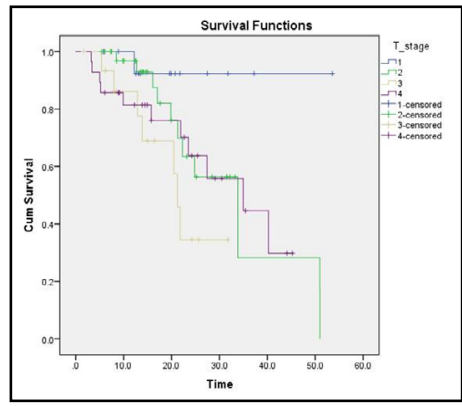

Figure 10. Kaplan-Meier overall survival curves for the $\mathbf{N}$ stage. There is a no significant difference between different $\mathbf{N}$ stages $(p=0.591)$.

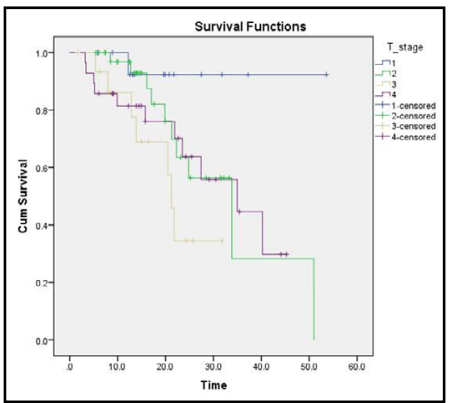

Figure 11. Kapalan -Meier overall survival for overall stage at diagnosis. There is no significant difference between the stages $(\mathrm{p}=0.224)$.

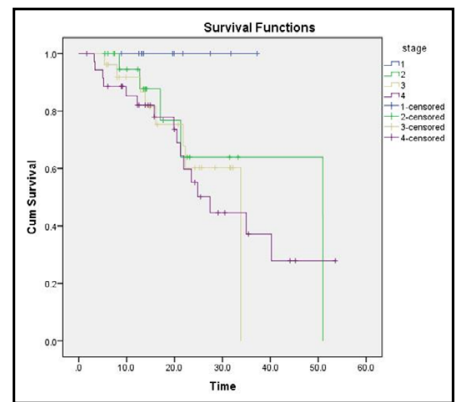


survival of 33.83 months and out of 21 patients with N2 status, $7(33.3 \%)$ are disease free while $14(66.7 \%)$ had recurrence of disease with a median disease free survival of 24.23 months. The overall median disease free survival was 24.83 months ( $\mathrm{p}$ value $=$ 0.381).

When overall stage was considered, out 11 patients with stage 1 disease, $9(81.8 \%)$ were disease free and $2(18.2 \%)$ had recurrence of disease with a median disease free survival of 37.23 months, out of 22 patients with stage 2 disease, 14 (63.6.\%) are alive while $8(36.4 \%)$ had recurrence of disease with a median disease free survival of 33.26 months and out of 26 patients with stage 3 disease, $16(61.5 \%)$ are alive while $10(38.5 \%)$ had recurrence of disease with a median disease free survival of 33.83 months and out of 36 patients with stage 4 disease, 14 (38.9\%) are disease free and $22(61.1 \%)$ had recurrence of disease with a median disease free survival of 21.93 months. The overall median disease free survival is 24.83 months.

The $\mathrm{p}$ value for the overall stage in Kaplan-Meier analysis for disease free survival was 0.479 which is not significant.

The $\mathrm{p}$ value for disease free survival when chi square test was done for the overall stage of disease was $0.044(p<0.05)$ and is statistically significant (Fig 12-14).

\section{Histological differentiation}

Overall and disease free survival: While comparing overall survival in 9 patients with histological differentiation of early SCC, 6 $(66.7 \%)$ are alive while $3(33.3 . \%)$ died with a median survival of 33.83 months, out of 68 patients with WDSCC, $47(69.1 \%)$ are alive while $21(30.9 \%)$ are dead with a median survival of 27.40 months, out of 11 patients with MDSCC, 10 (90.9\%) are alive while, $1(9.1 \%)$ is dead with a median survival of 40.23 months, out of 2 patients with PDSCC, both of them are dead with a median survival of 8.46 months. The overall median survival is 34.96 months $(\mathrm{p}$ value $=0.001)$.

While comparing disease free survival in 9 patients with histological differentiation of early SCC, $6(66.7 \%)$ are disease free while $3(33.3 . \%)$ had recurrence of disease with a median disease free survival of 33.83 months, out of 68 patients with WDSCC, 38 $(55.9 \%)$ are disease free while $30(44.1 \%)$ had recurrence of disease with a median disease free survival of 24.23 months, out of 11 patients with MDSCC, 7 (63.6\%) are disease free while, 8

Figure 12. Kaplan-Meier disease free survival curves for the $T$ stage. There is a no significant difference between different T stages $(p=0.381)$.

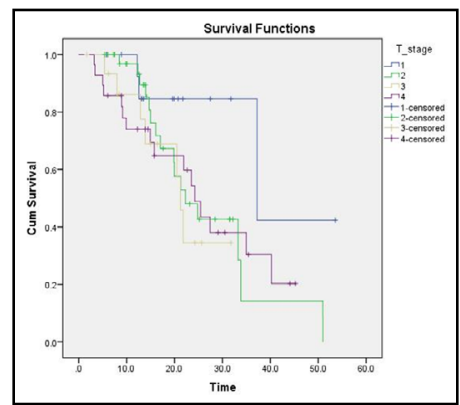

Figure 13. Kaplan-Meier disease free survival curves for the $\mathbf{N}$ stage. There is a no significant difference between different $\mathrm{N}$ stages $(\mathrm{p}=0.381)$.

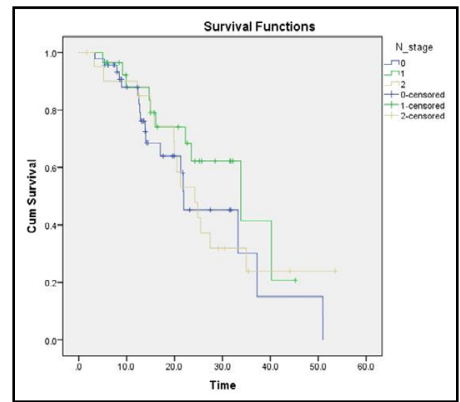

Figure 14. Kaplan-Meier disease free survival curves for overall stage. There is no significant difference between the stages $(\mathrm{p}=0.479)$.

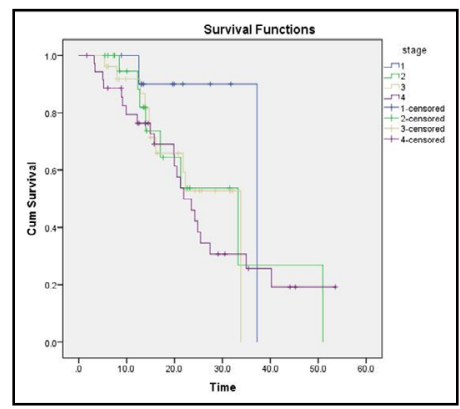


(36.4\%) have recurrence of disease with a median disease free survival of 40.23 months, out of 2 patients with PDSCC, both of them had recurrence of disease with a median disease free survival of 8.467 months. ( $\mathrm{p}$ value $=0.359$ in proportional analysis). The $\mathrm{p}$ value for histological grade in Kaplan-Meier analysis for overall survival and disease free survival is 0.001 and 0.021 respectively which is statistically significant (Fig 15 \& 16).

\section{Adjuvant therapy}

Overall survival: Out of 43 patients receiving adjuvant therapy $28(65.1 \%)$ are disease free while $15(24.9 \%)$ and out of $52 \mathrm{pa}-$ tients who did not receive adjuvant therapy, $38(73.1 \%)$ are alive and $14(26.9 \%)$ are dead.

Disease free survival: Out of 43 patients receiving adjuvant therapy $20(46.5 \%)$ are disease free while $23(53.5 \%)$ had recurrence of disease and out of 52 patients who did not receive adjuvant therapy, $33(63.5 \%)$ are disease free and $19(36.5 \%)$ had recurrence of disease.

The $\mathrm{p}$ value for overall survival and disease free survival in $\mathrm{Ka}$ plan-Meier analysis is 0.402 and 0.098 respectively and is statistically not significant.

\section{Discussion}

The total number of patients in our study was 95 . The male to female ratio is $1: 3$ with males more prone to OSCC than females in our study. The mean age at presentation in our study was $53 \pm$ 11.9. The mean follow up time in our study is 15 months (Inter quartile range 9.9 months to 24.8 months).

In many studies of Multivariate analysis, locoregional control, positive margins, vascular invasion, perineural invasion, extracapsular extension, and $\mathrm{T}$ classification has predicted overall and disease free survival [6].

In our study a significant association was found only between the stage of disease at presentation and the outcome we studied (DFS, OS). All the other associations between age, gender, subsite, histological variant of disease, adjuvant therapy administration, status of surgical margins, and disease free and overall survival were not found to be significant.

In our study the age, gender and subsite did not seem to be significant predictors of overall and disease-free survival for patients with OSCC like stage of the disease. The results are similar to other published studies in the literature. No survival difference was observed between those patients with carcinoma of the tongue and those with carcinoma of other subsites in the oral cavity [7]. From the results of the previous study it could be concurred that the site of presentation of carcinoma in the oral cavity (subsite) has less role in predicting the survival outcome of patients who are treated with multi-modal therapy and the results obtained in our study is also similar to the results of this study.

According to the American Joint Committee on Cancer the 5 -year actuarial rates of locoregional control by stage were: stage I, $100 \%$; stage II, $84 \%$; stage III, 78\%; and stage IV, 66\% (Hinerman 2004). Grade and stage are found to be independent factors in predicting survival in patients with oral squamous cell carcinoma $[2,8]$. Tumorswith high grade (grade 3) histology, regardless of $\mathrm{T}$ stage, had more propensity for regional metastasis. One study stated that achieving tumor-free margins in patients with poorly differentiated carcinomas (grade 3) versus other tumor grades was relatively difficult [8]. But in our study the $\mathrm{T}$ and $\mathrm{N}$ stage independently did not seem to predict disease free or overall survival but only the overall stage of the patient at the time of presentation (in chi square test) and histological grade (in KaplanMeier analysis) of the disease seemed to influence the disease free and overall survival.

Figure 15. Kaplan-Meier overall survival curves for histological grade. There is a significant difference in overall survival among different histological grades $(\mathrm{p}=\mathbf{0 . 0 0 1})$.

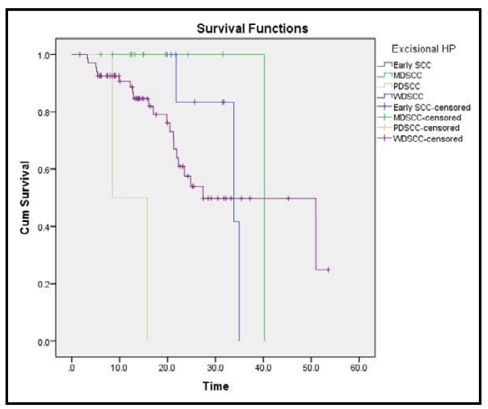

Figure 16. Kaplan-Meier disease free survival curves for histological grade. There is a significant difference in disease free survival among different histological grades $(\mathrm{p}=0.021)$.

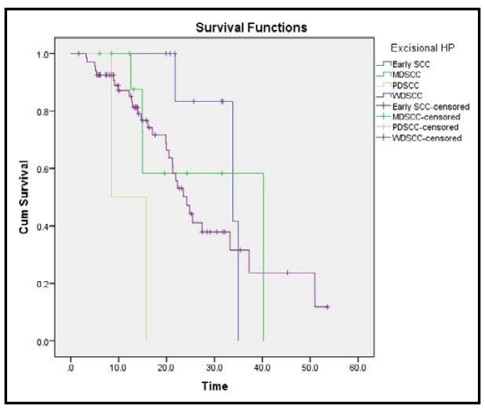


Intra-operatively the presence of a dysplastic margin is often unpredictable for a surgeon because of other variables that influence the presence or absence of dysplasia. The number one factor that leads to the presence of dysplasia is due the nature of cancer formation and progression. The concept of field cancerization is the cause for it. Clinically oral squamous cell carcinoma though found to be in a specific subsite, the entire epithelial surface of the upper GI tract is found to harbor genetic abnormalities and dysplastic lesions leading to a field effect, which is attributed to tobacco smoking, alcohol and areca nut and betel leaf consumption. Presence of widespread dysplasia surrounding the main tumor, prevents complete excision of the tumor and hence the only goal of a surgeon will be to prevent the presence of positive or close margin.

Tseng-Cheng Chen et al concludes in his study that the survival rates of the patients with OSCC who had dysplastic margin were significantly better than those with positive margin [9].

AS Jones et al in their study conclude that, The 5 year tumourspecific survival of patients with a positive margin was poorer by $12 \%$ than for those patients with a negative margin. Patients in their study with positive margins were tending to die of local recurrence [10].

Many studies have demonstrated the benefits of achieving negative resection margins in regards to local disease free survival and OS [11]. We could not demonstrate any significant survival benefit in our study. In our study the association between the surgical margin status and disease free and overall survival was not found to be significant.

Postoperative radiotherapy (RT) is given to act against microscopic deposits of cancer cells, which can cause locoregional recurrence if left unchecked [6].

The indications to give postoperative irradiation for carcinoma of the oral cavity are bone invasion or extension of tumor into the soft tissues of the neck (T4 primary); margins that are positive, close (V5 mm), contain CIS or dysplasia, or that were initially positive (but ultimately negative after re-resection;) perineural invasion; vascular space invasion; or multicentricity (scattered, discontinuous foci or islands of tumor adjacent to the primary site, making margins of resection uncertain), multiple positive lymph nodes or ECE $[6,12,13]$ which is very similar to the protocol being followed in our institute. In our study the administration of adjuvant therapy did not seem to predict disease free and overall survival.

\section{Conclusion}

Histological grade of the disease and stage at presentation seem to be important factors in determining disease free and overall survival stressing the importance of early detection and treatment of oral cancer to improve a patient's chance of success in terms of long term quality of life without recurrence.

\section{References}

[1]. Moore SR, Johnson NW, Pierce AM, Wilson DF. The epidemiology of mouth cancer: A review of global incidence.Oral Dis. 2000;6(2):65-74. PubmedPMID: 10702782 .

[2]. Kademani D, Bell RB, Bagheri S, Holmgren E, Dierks E, Potter B, et al. Prognostic factors in intraoral squamous cell carcinoma: The influence of histologic grade. J Oral MaxillofacSurg 2005;63(11):1599-605.PubmedPMID: 16243176.

[3]. McMahon J, O’Brien CJ, Pathak I, Hamill R, McNeil E, Hammersley N, et al. Influence of condition of surgical margins on local recurrence and disease-specific survival in oral and oropharyngeal cancer. $\mathrm{Br} \mathrm{J}$ Oral MaxillofacSurg2003;41(4):224-31.Pubmed PMID: 12946663.

[4]. Binahmed A, Nason RW, Abdoh AA. The clinical significance of the positive surgical margin in oral cancer. Oral Oncol 2007;43(8):780-4.Pubmed PMID: 17174145.

[5]. Margaret Brandwein-Gensler ;Miriam S. Teixeira;Carol Ming Lewis;Bryant Lee. Oral Squamous Cell Carcinoma ( OSCC ) 1997;29(2):140-6.

[6]. Hinerman RW, Mendenhall WM, Morris CG, Amdur RJ, Werning JW, Villaret DB. Postoperative irradiation for squamous cell carcinoma of the oral cavity: 35-Year experience. Head Neck 2004;26(11):984-94.Pubmed PMID: 15459927.

[7]. Bell RB, Kademani D, Homer L, Dierks EJ, Potter BE. Tongue Cancer: Is There a Difference in Survival Compared With Other Subsites in the Oral Cavity? J Oral MaxillofacSurg 2007;65(2):229-36.Pubmed PMID: 17236926.

[8]. Shiboski CH, Schmidt BL, Jordan RCK. Tongue and tonsil carcinoma: Increasing trends in the U.S. population ages 20-44 years. Cancer 2005;103(9):1843-9.Pubmed PMID: 15772957.

[9]. Chen TC, Chang HL, Yang TL, Lou PJ, Chang YL, Ko JY, Wang CP. Impact of dysplastic surgical margins for patients with oral squamous cell carcinoma. Oral Oncol. 2019 Oct;97:1-6. Pubmed PMID: 31421465.

[10]. Jones AS, Bin Hanafi Z, Nadapalan V, Roland NJ, Kinsella A, Helliwell TR. Do positive resection margins after ablative surgery for head and neck cancer adversely affect prognosis? A study of 352 patients with recurrent carcinoma following radiotherapy treated by salvage surgery. $\mathrm{Br} \mathrm{J}$ Cancer 1996;74(1):128-32.Pubmed PMID: 8679446.

[11]. Sieczka E, Datta R, Singh A, Loree T, Rigual N, Orner J, et al. Cancer of the buccal mucosa: Are margins and T-stage accurate predictors of local control? Am J Otolaryngol Head Neck Med Surg. 2001;22(6):395-9.Pubmed PMID: 11713724.

[12]. Lydiatt DD, Robbins KT, Byers RM, Wolf PF. Treatment of stage I and II oral tongue cancer Head Neck. 1993;15(4):308-12.Pubmed PMID: 8360052 .

[13]. Huang D, Johnson CR, Schmidt-Ullrich RK, Sismanis A, Neifeld JP, Weber J. Incompletely resected advanced squamous cell carcinoma of the head and neck: The effectiveness of adjuvant vs. salvage radiotherapy. RadiotherOncol 1992;24(2):87-93. Pubmed PMID: 1496147. 